\title{
Estudo Etnobotânico de plantas medicinais utilizadas pela Comunidade do Sisal no município de Catu, Bahia, Brasil
}

\author{
NETO, F.R.G. ${ }^{* 1}$; ALMEIDA, G.S.S.A. ${ }^{2}$ JESUS, N.G. ${ }^{3}$; FONSECA, M.R. ${ }^{4}$ \\ 1 Graduado em Ciências Biológicas pela Universidade do Estado da Bahia (UNEB) Campus II. Rodovia Alagoinhas/ \\ Salvador - BR 110 - KM 03 - Alagoinhas/BA, CEP 48040-210. (netinhorgn@hotmail.com). 2 Professora da \\ UNEB/ DCET/Campus II. Doutora em Botânica pela Universidade Federal de Viçosa, Curadora do Herbário \\ da Universidade do Estado da Bahia (HUNEB). ${ }^{3}$ Professor da UNEB/ DCET/Campus II, Rodovia, Mestre em \\ Ecologia pela Universidade Federal da Bahia- UFBA. ${ }^{4}$ Professor da UNEB/DCET/Campus II, Mestre em Botânica \\ pela Universidade Federal Rural de Pernambuco (UFRPE) e Doutor em Biologia Vegetal pela Universidade \\ Estadual de Campinas (UNICAMP).
}

\begin{abstract}
RESUMO: A utilização de plantas medicinais é instintiva nos animais, visto que alguns destes buscam raízes, cascas, folhas ou frutos na tentativa de resolver seus males. O homem, por sua vez, aprendeu a utilizar estas informações empiricamente para fazer uso destes vegetais. A medicina popular é uma importante alternativa, provavelmente, a mais usada para a cura de doenças por parte de populações indígenas, quilombolas ou rurais. A Etnobotânica, por sua vez, encarrega-se de estudar e interpretar essa relação dos homens com o mundo vegetal. Devido ao seu grande potencial biológico e cultural, o Brasil apresenta uma infinidade de conhecimentos tradicionais e espécies vegetais importantes, o que torna esse país uma grande fonte de pesquisa na área. Visando avaliar o conhecimento tradicional da população rural do Sisal, Catu/Bahia, relacionado ao uso de plantas medicinais, este trabalho iniciou-se em julho de 2009, constando de entrevistas semi-estruturadas e estruturadas, gravações, registros fotográficos, coleta do material botânico indicado nas entrevistas com os informantes, tratamento do material coletado e incorporação ao Herbário da Universidade do Estado da Bahia (HUNEB). Foram identificadas e coletadas 54 espécies distribuídas em 46 gêneros e 28 famílias, sendo Lamiacaeae e Asteraceae as mais representativas. A maioria das plantas é constituída de ervas e cultivada nos quintais dos moradores. O estudo revelou que a comunidade apresenta uma medicina popular bastante rica, com grande diversidade de espécies vegetais e usos por parte da população.
\end{abstract}

Palavras-chave: Etnobotânica, conhecimento tradicional, plantas medicinais, medicina popular.

ABSTRACT: Ethnobotanical study of medicinal plants used by the Sisal Community in the city of Catu, Bahia, Brazil. The use of medicinal plants is instinctive in animals, since some of these seek roots, bark, leaves or fruits in an attempt to cure their diseases. Man, in turn, learned to use this information on the use of these plants empirically. Folk medicine is an important alternative, probably the most used to cure diseases by indigenous peoples, Afro-descendants and rural populations. Ethnobotany, in turn, undertakes to study and interpret the relationship of men with the plant world. Because of its great biological and cultural potential, Brazil has a wealth of traditional knowledge and important plant species, what makes this country a great source of research in the area. To evaluate the traditional knowledge of the rural population of Sisal, city of Catu, state of Bahia, Brazil, related to use of medicinal plants, this work began in July 2009, consisting of semi-structured and structured interviews, recordings, photographs, collection of botanical material indicated in the interviews with informants, treatment of the collected material and incorporation into the Herbarium of the University of Bahia (HUNEB). We identified and collected 54 species in 46 genera and 28 families, being Asteraceae and Lamiacaeae the most representative. Most plants are herbs grown in the backyards of the residents. The study revealed that the community has a very rich folk medicine, with a great diversity of plant species and uses by the population.

Key words: Ethnobotany, traditional knowledge, medicinal plants, folk medicine. 


\section{INTRODUÇÃO}

Desde o inicio de sua existência o homem tem se relacionado com as plantas e animais. Para satisfazer suas necessidades, extraia recursos da natureza, garantindo sua sobrevivência e adaptação ao meio circundante. No curso de sua historia acumulou informações sobre o ambiente que o cerca e esse acervo baseou-se na observação dos fenômenos e características da natureza e na experimentação empírica desses recursos (PATZLAFF, 2007).

O uso das plantas medicinais é, ainda hoje, uma importante alternativa, para a cura de doenças por parte de populações indígenas, quilombolas ou das comunidades rurais. $O$ conhecimento tradicional foi transmitido ao longo de vários anos, de geração a geração, passando de pai para filho, geralmente de forma oral. A utilização de plantas medicinais por populações rurais é orientada por uma série de conhecimentos acumulados mediante a relação direta dos seus membros com o meio ambiente e da difusão de informações tendo como influência o uso tradicional transmitido oralmente entre diferentes gerações (MOREIRA et al. 2002).

Com o passar dos anos, o surgimento de novas tecnologias e o aumento de ações antrópicas nos ambientes naturais, fez com que a medicina popular sofresse uma diminuição de seu uso por parte da sociedade. A evidente descaracterização das comunidades tradicionais, acompanhada da destruição de habitats e da inserção de novos elementos culturais, põe em risco um grande acervo de conhecimentos empíricos e um patrimônio genético de valor inestimável para as futuras gerações, (PIRES et al. 2009).
As comunidades tradicionais, em função da forte influência do meio natural, apresentam modos de vida e cultura diferenciadas. Seus hábitos estão diretamente submetidos aos ciclos naturais e a forma como apreendem a realidade e a natureza é baseada não só em experiência e racionalidade, mas em valores, símbolos, crenças e mitos (MONTELES \& PINHEIRO, 2007). Dessa forma tais comunidades tornam-se o principal objeto de estudo Etnobotânica.

A Etnobotânica pode ser definida como a ciência que estuda as sociedades humanas, passadas e presentes, e suas interações ecológicas, genéticas, evolutivas, simbólicas e culturais com as plantas (FONSECA-KRUEL \& PEIXOTO, 2004). É uma ciência multidisciplinar que envolve diversas áreas como a botânica, a antropologia, a farmacologia, a fitoquímica e a medicina.

Atualmente a comunidade cientifica tem despertado grande interesse em realizar estudos etnobotânicos, principalmente relacionados à categoria das plantas medicinais, objetivando descobrir novas plantas empregadas na medicina popular bem como seus princípios ativos, além de preservar o conhecimento tradicional.

O presente trabalho foi realizado com o objetivo de analisar o conhecimento da comunidade rural do Sisal relacionado ao uso tradicional de plantas medicinais.

\section{MATERIAL E MÉTODO}

O trabalho foi realizado na Comunidade do Sisal no município de Catu-Ba, situado a

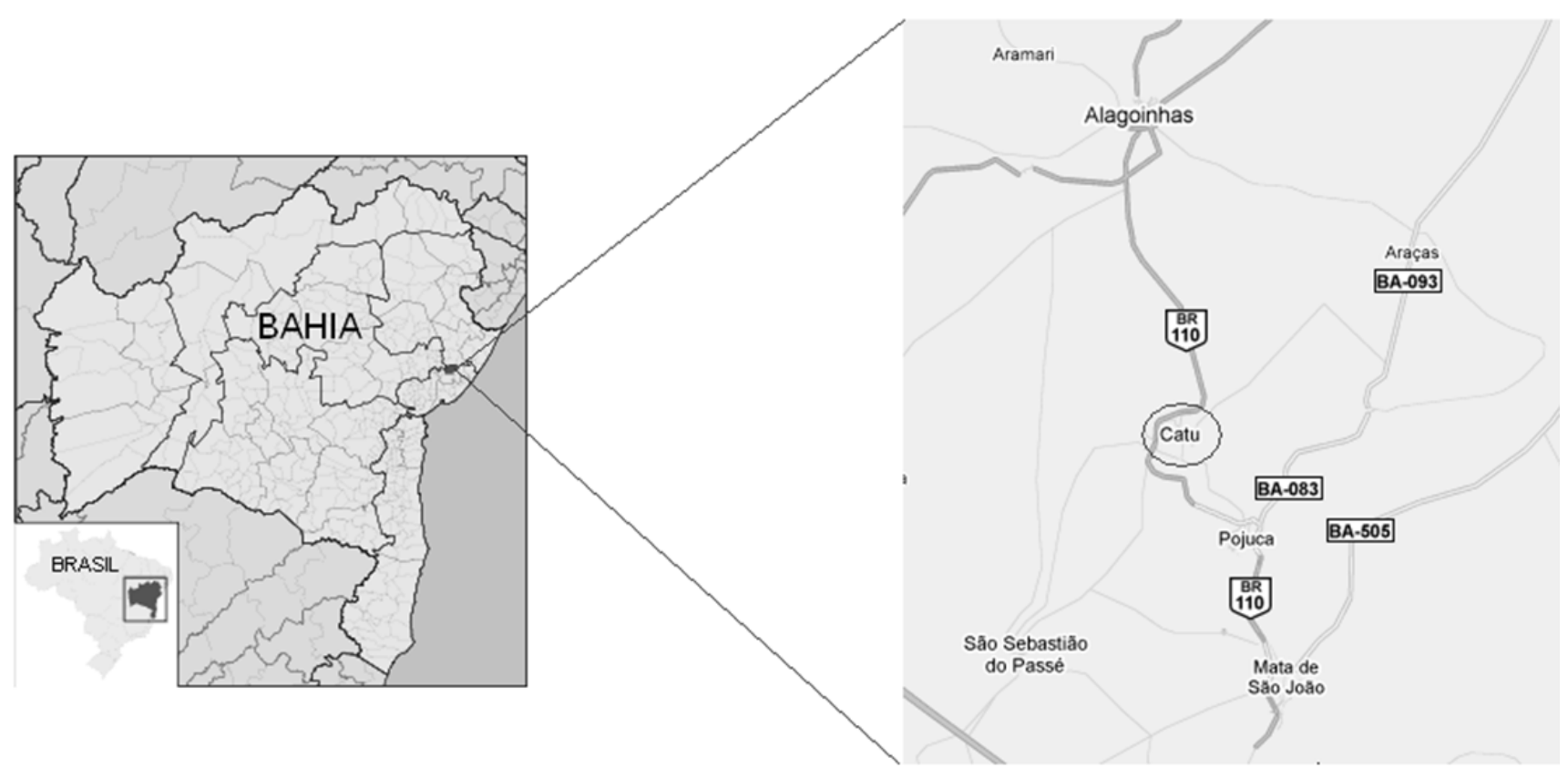

FIGURA 1. Localização da área de estudo. Fonte: maps.google.com.br, 2010. 
aproximadamente $78 \mathrm{~km}$ de Salvador (Figura 1), nas coordenadas $12^{\circ} 21^{\prime} \mathrm{S}, 38^{\circ} 23^{\prime} \mathrm{W}$ e altitude de $177 \mathrm{~m}$. A pluviosidade media anual é de 1200 a 1600 mm, sendo que o período chuvoso ocorre entre os meses de abril e julho. A vegetação da região é do tipo Floresta Ombrófila Densa. A Cidade esta localizada na Bacia Hidrográfica do Rio Pojuca (CEI, 1994).

A comunidade do Sisal dista aproximadamente $6 \mathrm{~km}$ da zona urbana da cidade de Catu, localizada nas coordenadas de $12^{\circ} 19^{\prime}$ $39,4^{\prime \prime}$ S e $38^{\circ} 24^{\prime} 52,8^{\prime \prime} \mathrm{W}$.

Na primeira etapa foram realizadas visitas de reconhecimento da área e aproximação com a população residente, para reconhecimento sóciocultural e identificação dos informantes-chave.

A segunda etapa do trabalho envolveu a aplicação de questionários semiestruturados utilizados como roteiro nas conversas com os informantes-chave, visando obter as informações referentes às espécies e a parte utilizada das plantas, as formas de uso e as enfermidades para as quais eram indicadas. As conversas foram gravadas mediante a autorização do entrevistado. O critério de escolha dos informantes considerou o tempo de residência na comunidade e a idade, entendendo que os mais idosos e os que moram ha mais tempo na comunidade sejam os maiores detentores do conhecimento tradicional, além do critério de ter sido indicado pelo conjunto da população.

A terceira etapa envolveu a coleta e o registro fotográfico do material botânico no período de julho de 2009 a maio de 2010. Sendo essas coletas acompanhadas pelos informantes-chave.

A quarta etapa envolveu a herborização do material coletado e a incorporação no Herbário da Universidade do Estado da Bahia - HUNEB. A herborização seguiu as técnicas de Fidalgo \& Bononi (1984). Para a identificação do material coletado foram realizadas consultas à literatura botânica, comparação com o material existente no HUNEB, e consultas a especialistas de determinados grupos botânicos. As espécies de plantas identificadas foram organizadas segundo o sistema APG II.

Os dados etnobotânicos obtidos foram organizados em um banco de dados a partir do programa Excel contendo as seguintes informações: nome popular, nome cientifico; família, parte utilizada, uso, preparo, local de extração; informante; número de tombo e número de citações. A categorização das doenças seguiu a literatura etnobotânica consultada, dentre elas Monteles \& Pinheiro (2007). Os dados foram utilizados para confecção dos gráficos apresentados.

Segundo Amorozo (2006), a porcentagem de concordância quanto aos usos principais (CUP) foi calculada para determinar a importância relativa das espécies citadas na comunidade. Seu valor foi determinado baseando-se no seguinte cálculo: CUP $=($ ICUP / ICUE $) \times 100$, onde ICUP representa o número de informantes que citaram os usos principais e ICUE o número de informantes que citaram o uso da espécie. Aqui foram consideradas as espécies citadas por três ou mais informantes.

As entrevistas gravadas foram transcritas e analisadas visando extrair os dados qualitativos, importantes para fundamentação dos resultados.

Para a realização desta pesquisa, o projeto foi avaliado pelo Comitê de Ética em Pesquisa (CEP) da Universidade do Estado da Bahia, sendo devidamente aprovado de acordo com o parecer de número 0603100013670, adequando-se aos critérios estabelecidos pelo Código de Ética em Pesquisa - Resolução CNS n 196/96.

\section{RESULTADOS E DISCUSSÕES}

A comunidade tem uma população de aproximadamente 60 moradores, com uma grande parcela $(50 \%)$ de jovens abaixo de 20 anos. Pelo que pode ser observado é comum as mulheres casarem ou terem filhos muito cedo, refletindo no número elevado de crianças e adolescentes na comunidade.

Dos seis informantes selecionados, quatro eram do sexo masculino e dois do sexo feminino, com idades variando entre 35 a 55 anos. Três informantes moram na comunidade há mais de 31 anos, dois, moram há 16 e 20 anos, e apenas um mora há, aproximadamente, 12 anos. Cinco possuem apenas o ensino fundamental e um afirmou nunca ter ido à escola, realidade comum em comunidades rurais, onde geralmente as crianças começam a trabalhar muito cedo nas roças, sob orientação dos pais, não tendo tempo suficiente para estudar. Este fato foi também observado por Amorozo (2002) em um trabalho realizado no Município de Santo Antonio do Leverger no estado de Mato Grosso, local de baixo nível de escolaridade devido ao fato da maior parte dos moradores estar ou ter estado ligado à atividades agrícolas ou de pesca.

Foram citadas e devidamente coletadas nos quintais e em áreas ruderais indicadas pelos informantes-chave, 54 espécies botânicas distribuídas em 46 gêneros e 28 famílias (Tabela 1).

Lamiaceae foi a família mais representada, com 7 espécies, seguida de Asteraceae, com 5 espécies. Resultado semelhante ao encontrado por Moreira et al. (2002).

A família Lamiaceae destaca-se por ser bastante representativa nos estudos referentes a plantas medicinais, este grupo botânico possui vários representantes com propriedades terapêuticas comprovadas (MOSCA \& LOIOLA, 2009), a exemplo do Ocimum gratissimum L., cujo óleo essencial contém eugenol (SARTÓRIO et al., 2000). A família

Rev. Bras. Pl. Med., Campinas, v.16, n.4, p.856-865, 2014. 
TABELA 1. Lista das espécies amostradas na Comunidade do Sisal, Catu, Bahia

\begin{tabular}{|c|c|c|c|c|c|c|}
\hline Família/Nome científico & N.P. & P.U. & Indicação & $\mathrm{E} / \mathrm{C}$ & HAB. & N.T. \\
\hline \multicolumn{7}{|l|}{ Adoxaceae } \\
\hline Sambucus australis Cham. \& Schltdl. & Sabugueiro & $\mathrm{FI}$, Fo & Gripe & Qu & Arv & 12815 \\
\hline \multicolumn{7}{|l|}{ Amaranthaceae } \\
\hline Pfaffia glomerata (Spreng.) Pedersen & Anador & Fo & Dor de cabeça & Qu & Sarb & 12817 \\
\hline \multicolumn{7}{|l|}{ Anacardiaceae } \\
\hline Schinus terebinthifolius Raddi & Aroeira & Fo & $\begin{array}{c}\text { Inflamação e } \\
\text { doenças de pele }\end{array}$ & Qu & Arv & 12858 \\
\hline \multicolumn{7}{|l|}{ Annonaceae } \\
\hline Annona sp & Jaca de pobre & Fo & Febre & Qu & Arv & 12900 \\
\hline \multicolumn{7}{|l|}{ Apocynaceae } \\
\hline Thevetia peruviana K.Schum. & Tontice & $\mathrm{Se}$ & Tontura & A.R & Arv & 12907 \\
\hline \multicolumn{7}{|l|}{ Asteraceae } \\
\hline Acanthospermum hispidum DC. & Juiz de Paz & $\mathrm{Ra}$ & Inflamação & Qu & Erv & 12822 \\
\hline Bidens pilosa $L$. & Picão & $\mathrm{Ra}$ & Emagrecedor & Qu & Erv & 12901 \\
\hline Sphagneticola trilobata (L.) Pruski & Mal-me-quer & Fo, Ra & $\begin{array}{c}\text { Tosse, } \\
\text { Tuberculose }\end{array}$ & Qu & Erv & 12864 \\
\hline Verbesina macrophylla S.F. Blake & $\begin{array}{l}\text { Assa peixe } \\
\text { branco }\end{array}$ & Fo & Gripe & Qu & Sarb & 12916 \\
\hline Vernonia bahiensis Toledo & Alumã & Fo & Dor de barriga & Qu & Arv & 12865 \\
\hline \multicolumn{7}{|l|}{ Boraginaceae } \\
\hline Heliotropium indicum L. & Crista de Galo & $\mathrm{Ra}$ & $\begin{array}{l}\text { Dor de barriga e } \\
\text { dor de dente }\end{array}$ & A.R & Sarb & 12808 \\
\hline $\begin{array}{l}\text { Cordia curassavica (Jacq.) Roem. \& } \\
\text { Schult. }\end{array}$ & Maria Preta & Fo & Sinusite, gripe & A.R & Sarb & 12810 \\
\hline \multicolumn{7}{|l|}{ Burseraceae } \\
\hline $\begin{array}{l}\text { Commiphora leptophloeos (Mart.) } \\
\text { J.B.Gillett }\end{array}$ & Umburana & Fo & Dor de barriga, & Qu & Arv & 12912 \\
\hline Protium heptaphyllum Marchand & Améscara & $\begin{array}{l}\mathrm{Re}, \mathrm{Fo} \\
\mathrm{Fr}\end{array}$ & $\begin{array}{c}\text { Sinusite, gastrite, } \\
\text { dor de estomago, } \\
\text { dor de cabeça }\end{array}$ & A.R & Arv & 12866 \\
\hline \multicolumn{7}{|l|}{ Caricaceae } \\
\hline Carica papaya L. & Mamão macho & Fo & Gastrite & Qu & Arv & 12903 \\
\hline \multicolumn{7}{|l|}{ Chenopodiaceae } \\
\hline Chenopodium ambrosioides L. & Mastruz & Fo & Verminose & Qu & Erv & 12813 \\
\hline \multicolumn{7}{|l|}{ Convovulaceae } \\
\hline Ipomoea asarifolia Roem. \& Schult. & Batata de boi & P.I & $\begin{array}{c}\text { Dor de dente } \\
\text { (Antiinflamatório) }\end{array}$ & A.R & Erv & 12910 \\
\hline \multicolumn{7}{|l|}{ Clusiaceae } \\
\hline Vismia guianensis (Aubl.) Choisy & Capianga & Fo & Aborto & A.R & Arb & 12906 \\
\hline \multicolumn{7}{|l|}{ Cucurbitaceae } \\
\hline Momordica charantia L. & $\begin{array}{l}\text { Melão de São } \\
\text { Caetano }\end{array}$ & Fo & $\begin{array}{l}\text { Verminose, } \\
\text { indigestão }\end{array}$ & A.R & Therb & 12809 \\
\hline
\end{tabular}


TABELA 1. Lista das espécies amostradas na Comunidade do Sisal, Catu, Bahia

...continuação

\begin{tabular}{|c|c|c|c|c|c|c|}
\hline \multicolumn{7}{|l|}{ Cyperaceae } \\
\hline Rhynchospora nervosa Boeckeler & Capim estrela & P.I & Gripe & A.R & Erv & 12909 \\
\hline \multicolumn{7}{|l|}{ Euphorbiaceae } \\
\hline Cnidoscolus urens (L.) Arthur & Cansanção & Ra, P.I & Gripe & A.R & Erv & 12908 \\
\hline Euphorbia tirucalli L. & Gravetinho & La & $\begin{array}{l}\text { Câncer, úlcera, } \\
\text { gastrite }\end{array}$ & Qu & Arv & 12859 \\
\hline Croton campestris A. St. -Hil. & Velame & Fo & Constipação & A.R & Sarb & 12898 \\
\hline Phyllanthus niruri L. & Quebra pedra & $\mathrm{Ra}$ & Cálculo renal & Qu & Erv & 12826 \\
\hline \multicolumn{7}{|l|}{ Fabaceae } \\
\hline $\begin{array}{l}\text { Abarema cochliacarpos (Gomes) } \\
\text { Barneby \& J.W. Grimes }\end{array}$ & Babatenã & Ec & Inflamação & A.R & Arv & 12818 \\
\hline Caesalpinia pulcherrima (L.) Sw. & $\begin{array}{l}\text { Maravilha } \\
\text { amarela }\end{array}$ & Fo & Conjuntivite & A.R & Arb & 12915 \\
\hline Senna occidentalis (L.) Link & Fedegoso & $\mathrm{Ra}$ & Gripe & Qu & Erv & 12814 \\
\hline Zornia gemella Vogel & Arrozinho & P.I & Problemas renais & Qu & Erv & 12863 \\
\hline \multicolumn{7}{|l|}{ Lamiaceae } \\
\hline Hyptis sp. & Alfazema & Fo & $\begin{array}{l}\text { Dor de cabeça, } \\
\text { cólica }\end{array}$ & Qu & Sarb & 12856 \\
\hline Ocimum gratissimum L. & Quioio & Fo & $\begin{array}{l}\text { Pressão alta, } \\
\text { moleza no corpo } \\
\text { (indisposição). }\end{array}$ & Qu & Sarb & 12821 \\
\hline Ocimum micranthum Willd. & Alfavaca & Fo & Gripe & Qu & Sarb & 12812 \\
\hline Ocimum sp. & Manjericão & Fo & Dor de ouvido & Qu & Erv & 12904 \\
\hline Plectranthus amboinicus (Lour.) Spreng. & Hortelã graúda & Fo & Gripe, indigestão & Qu & Erv & 12918 \\
\hline Plectranthus barbatus Andrews & $\begin{array}{c}\text { Tapete do } \\
\text { oxalá }\end{array}$ & Fo & $\begin{array}{c}\text { Dores musculares, } \\
\text { indigestão }\end{array}$ & Qu & Erv & 12811 \\
\hline Plectranthus sp. & Boldo & Fo & Dor de barriga & Qu & Erv & 12902 \\
\hline \multicolumn{7}{|l|}{ Malpighiaceae } \\
\hline Malpighia glabra L. & Acerola & Fo & Gripe & Qu & Arv & 12861 \\
\hline \multicolumn{7}{|l|}{ Malvaceae } \\
\hline Gossypium herbaceum L. & Algodão & Fo, FI & $\begin{array}{c}\text { Inflamação, } \\
\text { mulher com } \\
\text { dor na barriga, dor } \\
\text { de cabeça }\end{array}$ & Qu & Arv & 12820 \\
\hline Pavonia cancellata (L.) Cav. & Escorredeira & P.I & Tratamento capilar & A.R & Erv & 12896 \\
\hline Sida linifolia Cav. & Língua de tatu & Fo & Furúnculo & A.R & Erv & 12897 \\
\hline \multicolumn{7}{|l|}{ Myrtaceae } \\
\hline Eugenia uniflora L. & Pitanga & Fo & Gripe & Qu & Arv & 12919 \\
\hline Psidium guajava $\mathrm{L}$. & Goiaba Branca & Fo & Dor de barriga & Qu & Arv & 12914 \\
\hline Psidium sp. & Araçá & Fo & Dor de barriga & A.R & Arv & 12825 \\
\hline
\end{tabular}


TABELA 1. Lista das espécies amostradas na Comunidade do Sisal, Catu, Bahia

...continuação

\begin{tabular}{|c|c|c|c|c|c|c|}
\hline \multicolumn{7}{|l|}{ Phytolacaceae } \\
\hline Petiveria alliacea L. & Guiné & Fo & $\begin{array}{c}\text { Tirar descarrego, } \\
\text { dor de cabeça }\end{array}$ & Qu & Sarb & 12913 \\
\hline \multicolumn{7}{|l|}{ Poaceae } \\
\hline Cymbopogon citratus Stapf & Capim santo & Fo & Pressão alta & Qu & Erv & 12905 \\
\hline Cymbopogon densiflorus Stapf & $\begin{array}{l}\text { Capim } \\
\text { Caboclo }\end{array}$ & Fo & Dor de barriga & Qu & Erv & 12816 \\
\hline \multicolumn{7}{|l|}{ Rubiaceae } \\
\hline Spermacoce verticillata L. & Carqueja & Ra, P.I & $\begin{array}{l}\text { Dor de barriga, } \\
\text { primeira dentição, } \\
\text { emagrecedor }\end{array}$ & Qu & Erv & 12819 \\
\hline \multicolumn{7}{|l|}{ Rutaceae } \\
\hline Citrus aurantium L. & Laranjeira & Fo & Gripe & Qu & Arv & 12824 \\
\hline \multicolumn{7}{|l|}{ Rutaceae } \\
\hline Ertela trifolia Kuntze & $\begin{array}{c}\text { Alfavaca de } \\
\text { cobra }\end{array}$ & Fo & $\begin{array}{l}\text { "Antipotente" } \\
\text { (causa impotência } \\
\text { sexual) }\end{array}$ & Qu & Sarb & 12854 \\
\hline \multicolumn{7}{|l|}{ Scrophulariaceae } \\
\hline Scoparia dulcis L. & Vassourinha & Fo & Dor de barriga & Qu & Arb & 12857 \\
\hline \multicolumn{7}{|l|}{ Solanaceae } \\
\hline Cestrum laevigatum Schltdl. & Corana & Fo. & Cefaléia & A.R. & Arb. & 12917 \\
\hline Solanum palinacanthum Dunal & Bobó & Fo & $\begin{array}{c}\text { Gripe, cólica } \\
\text { menstrual }\end{array}$ & A.R & Sarb & 12911 \\
\hline \multicolumn{7}{|l|}{ Verbenaceae } \\
\hline $\begin{array}{l}\text { Lippia alba (Mill.)N.E.Br. ex Britton \& } \\
\text { P. Wilson }\end{array}$ & Erva cidreira & $\mathrm{Ca}, \mathrm{Fo}$ & $\begin{array}{l}\text { Dor de barriga, } \\
\text { problema de } \\
\text { pressão arterial }\end{array}$ & Qu & Sarb & 12823 \\
\hline \multicolumn{7}{|l|}{ Violaceae } \\
\hline Hybanthus arenarius Ule & $\begin{array}{l}\text { Purga do } \\
\text { campo } 2\end{array}$ & $\mathrm{Ra}$ & $\begin{array}{c}\text { Gripe, } \\
\text { expectorante }\end{array}$ & Qu & Erv & 12862 \\
\hline Hybanthus calceolaria (L.) Oken & $\begin{array}{l}\text { Purga do } \\
\text { Campo } 1\end{array}$ & $\mathrm{Ra}$ & Diarréia & Qu & Erv & 12855 \\
\hline
\end{tabular}

Legenda: P.U.= parte utilizada; N.P.= nome popular; N.T.= número de tombo; Fo= folha; Ra=raiz; La=látex; P.I planta inteira; Ec=entrecasca; $\mathrm{Fr}=$ fruto; $\mathrm{Re}=$ resina; $\mathrm{Se}=$ semente; $\mathrm{Fl}=$ =flor; $\mathrm{Ca}=$ caule; $\mathrm{E} / \mathrm{C}=$ extração/coleta; Qu=quintal; $\mathrm{A} . \mathrm{R}=a$ rea ruderal; $\mathrm{HAB}=$ hábito; $E r v=$ erva; therb= trepadeira herbácea; Arb=Arbusto, Sarb=subarbusto; Arv= árvore.

Asteraceae é bastante estudada, também devido aos promissores compostos químicos produzidos por este grupo (ARAUJO et al. 2008). Bahia (1979) já citava o alumã (Vernonia bahiensis Tol.) com propriedade amargo-tônica, diurética e estomacal, Sartório et al. (2000) cita a espécie Picão, (Bidens pilosa L.) com princípios ativos comprovados, tais como o ácido salicílico, taninos, limoneno, candineno, timol, a-pineno e a-feladreno, sais de potássio, cálcio e fósforo.
Com relação aos gêneros, os mais representativos foram Ocimum e Plectranthus, ambos com três espécies, seguido de Psidium, Cymbopogon e Hybantthus com dois representantes cada.

A maioria das plantas utilizadas é de hábito herbáceo, $39 \%$, as quais são mais fáceis de serem cultivadas em Quintais e Hortas, seguidas por espécies arbóreas, 30\%, conforme representado na Figura 2. Resultado semelhante ao encontrado 
por Amorozo et al. (2006) em trabalho realizado em comunidades rurais de mata atlântica (Marambaia e Camboinha), em Itacaré-BA, Brasil, onde as ervas também foram as plantas predominantes.

Amaioria das plantas utilizadas é proveniente do cultivo em quintais, totalizando 37 espécies, o que equivale à, aproximadamente, $68 \%$ do estoque, enquanto as demais são coletadas em áreas ruderais, correspondendo à, aproximadamente, 32 $\%$ do estoque ou à 17 espécies de plantas.

Geralmente a população não faz coletas em áreas de mata, nem vai à feira em busca das plantas medicinais. Esse fato revela a eficiente relação dos moradores com os recursos naturais, cultivando as espécies de interesse medicinal próximo das residências.

As famílias compartilham os conhecimentos, de forma que os moradores também buscam as plantas cultivadas pelos vizinhos, ou consultam o conhecimento do outro. Esse comportamento também foi observado por Arnous et al. (2005) no município de Datas- MG, onde foi constatado que $78,5 \%$ das pessoas possuem o hábito de cultivar as plantas medicinais em seus quintais e jardins e $38,2 \%$ também adquirem nos quintais dos vizinhos e amigos.

Os tipos de afecções que obtiveram os maiores números de espécies citadas estão relacionados aos problemas do sistema respiratório e aos males ligados ao sistema digestivo, ambos com 16 citações, conforme representado na Figura 3. Resultado semelhante também foi encontrado por Monteles \& Pinheiro (2007) em um estudo sobre Plantas medicinais no Quilombo Sangrador (MA).

A espécie mais citada e com maior índice de concordância foi o Boldo (Plectrantus sp), com quatro citações e CUP igual a 100\%, revelando que esta espécie é a mais relevante do acervo medicinal da comunidade, a qual é utilizada pelos moradores para dor de barriga, podendo sugerir uma real efetividade no tratamento das doenças e sintomas associados ao sistema digestivo .

A folha foi a parte mais indicada, representando $61 \%$ das citações, seguida da raiz com 16\%, conforme representado na Figura 4. Resultado semelhante foi encontrado em um estudo acerca do uso e diversidade de plantas medicinais no Quilombo Olho D'água dos Pires no município de Esperantina (PI) feito por Franco \& Barros (2006), onde o uso da folha também predomina.

Foram encontradas diversas formas de preparo, dentre elas, o emplastro, defumador, infusão, maceração, xarope, banho, e a decocção, sendo este último o mais representativo com 29 citações (Figura 5).

Foram também encontradas outras formas de uso ou preparo representado pelo item Outros* da figura 5. Da planta tontice (Thevetia peruviana Pers) é feito um colar com a semente, usado para curar a tontura. Do gravetinho (Euphorbia tirucalli L.) extrai-se o látex, misturando com água, sendo a mistura, posteriormente ingerida.

A fala de alguns moradores também explica outras formas de preparo:

"O algodão, quando você ta com dor de cabeça, ela ta forte mesmo, aí você vai, tira a folha dele, coloca no ar do fogo, quando ela estiver morninha, você coloca na testa, amarra um pano e deixa ele lá por algum tempo" (J.J, 41 anos);

"Usa a folha e também a maçãzinha que ele bota, que faz o algodãozinho. Pra menino pequeno. Se tiver um menino molinho, pequeno e ele tiver

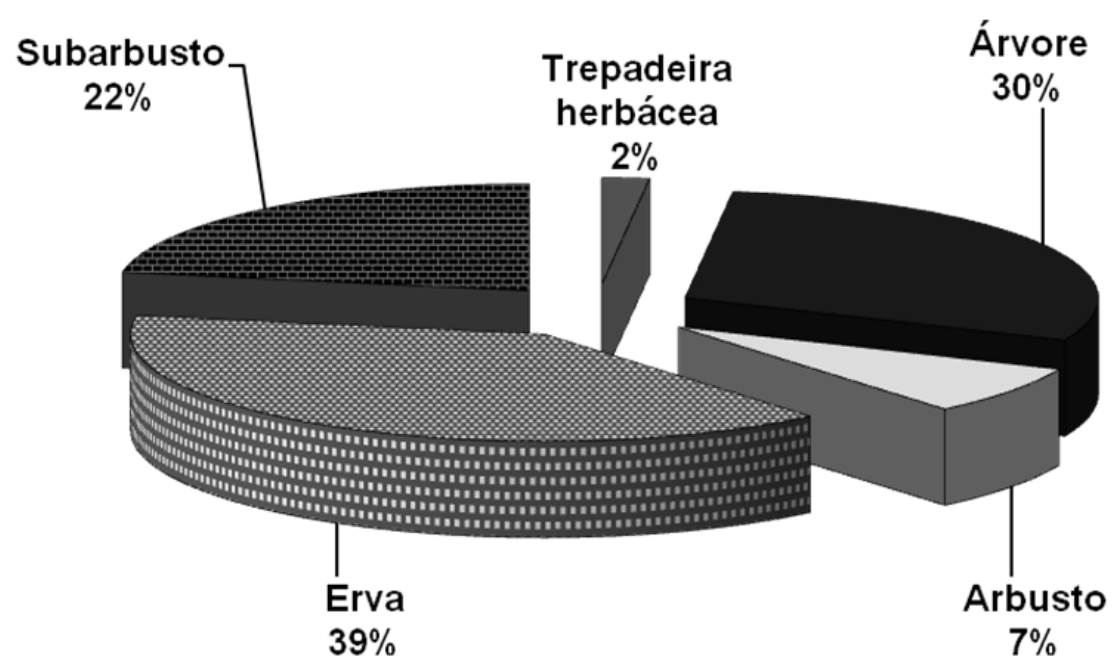

FIGURA 2. Representação gráfica dos hábitos das espécies coletadas na comunidade do Sisal, Catu, Bahia. 


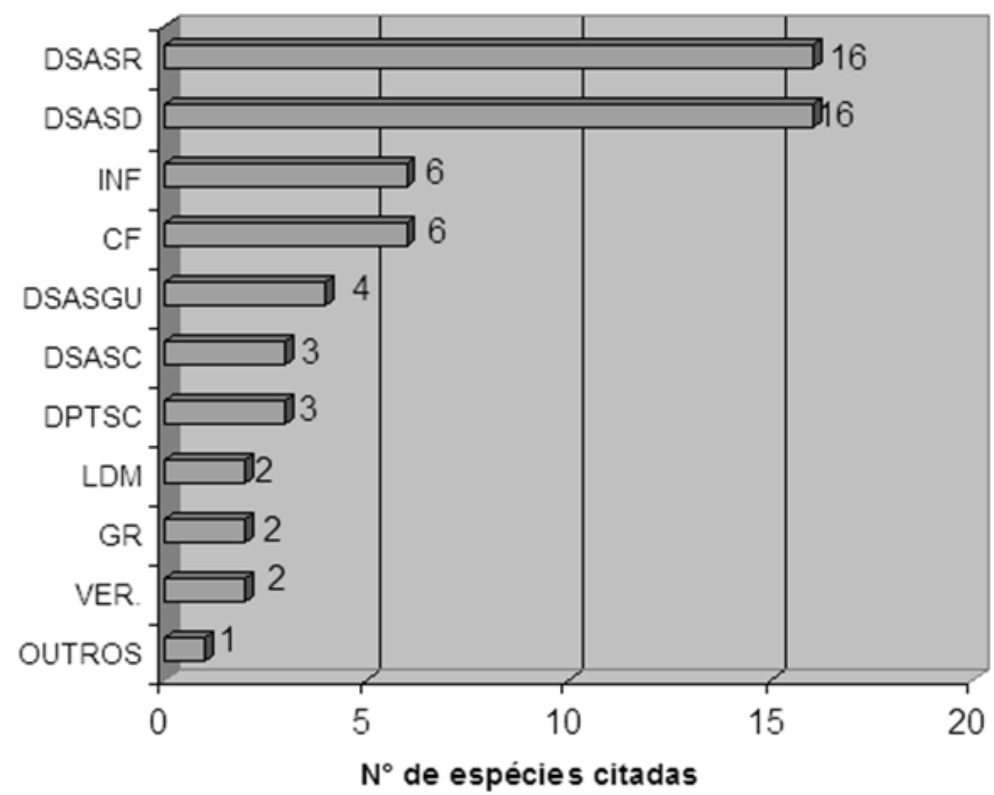

Legenda: $D S A S R=$ doença e sintomas associadas ao sistema respiratório; $D S A S D=$ doenças e sintomas associadas ao sistema digestivo; INF=infecções; $C F=$ cefaleia; $D S A S G U=$ doenças e sintomas associados ao sistema genito-urinário; $D S A S C=$ doenças e sintomas associados ao sistema circulatório; DPTSC=doenças de pele e do tecido subcutâneo; LDM=lesões e dores musculares; GR=gravidez; VER=verminoses; Outros=outros tipos de sintomas e doenças não enquadrados nos itens anteriores.

FIGURA 3. Representação gráfica das doenças e sintomas citados pela comunidade do Sisal, Catu, Bahia.

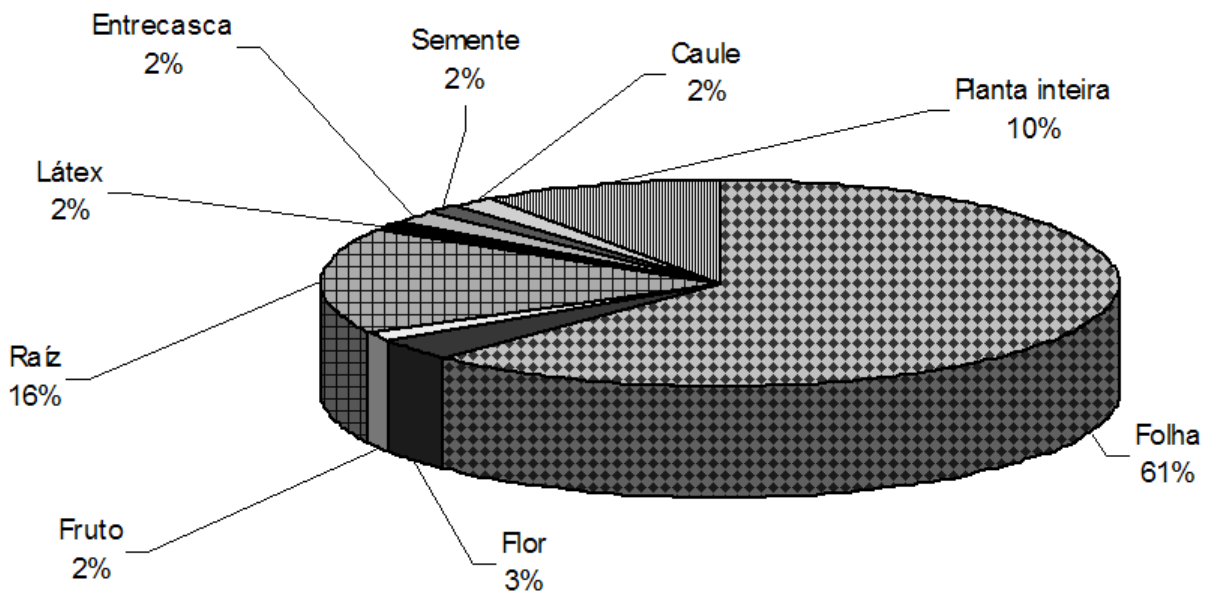

FIGURA 4. Representação gráfica das partes das plantas utilizadas pelos informantes da comunidade do Sisal, Catu, Bahia.

com dor de barriga, a gente pega aquela maçãzinha dele, bota assim dentro do "burralho", na cinza, aí quando ela começar a resinar, a gente aperta que ela cai aquelas gotinhas amarelinhas, que nem a dipirona, dentro da água fervida, aí a gente esfria e da ao menino pra beber" (M.P.J, 43 anos).

Um grande problema visualizado na comunidade é a questão da manutenção do conhecimento relacionado ao uso das plantas medicinais, alguns fatores têm interferido na sua perpetuação. Observa-se que a influência da medicina alopática tem diminuído o valor da medicina popular por parte da comunidade estudada. Isto se deve, principalmente, ao fato de que a comunidade não dispõe de escolas; dessa forma, a maioria dos jovens estudam em instituições da área urbana. Nesses ambientes, assimilam muitas informações 


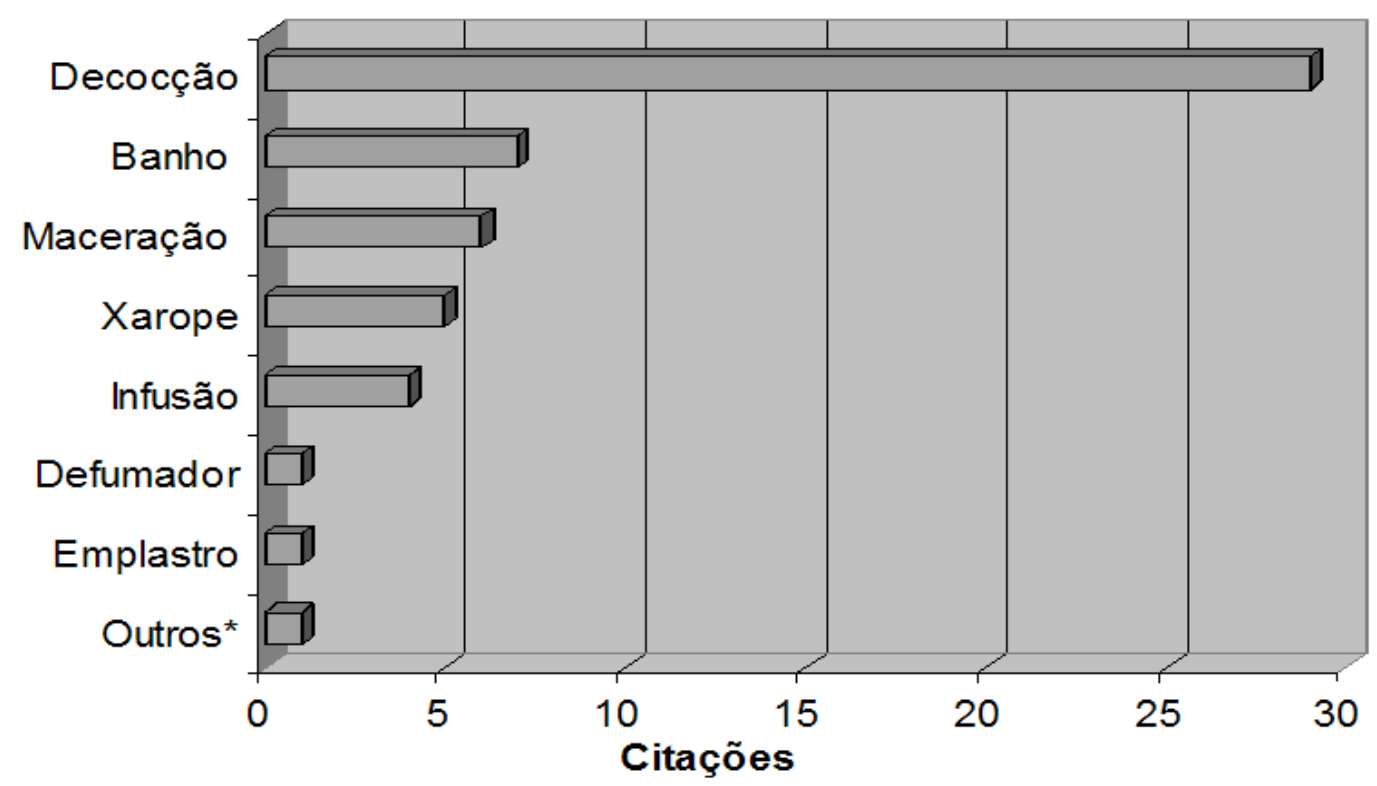

FIGURA 5. Representação gráfica das formas de preparo das plantas medicinais

relacionadas à tecnologia, estudos e descobertas científicas, o que torna, para eles, cada vez menos importante os ensinamentos dos seus pais e avós, principalmente sobre as plantas medicinais. Sendo assim, o uso dos medicamentos sintéticos vendidos nas farmácias torna-se mais confiável, tomando um valor maior dentro da comunidade.

A partir dos relatos dos moradores, pode-se observar esse problema. Quando questionados se existia alguma doença em que eles preferencialmente visitavam o médico, cinco deles responderam que sim, o relato de dois informantes a seguir pode esclarecer o que eles pensam a respeito disso:

"O pessoal tá com a cabeça doendo, você sabe que é uma dor de cabeça, se a pressão sobe, você sente no corpo que sua pressão ta alta. Ai tem a base, você vai usar. Desde quando já foi medicado né. As vezes no caso, pra saber, a gente não pode tomar sem primeiro ir ao medico a primeira vista" (J.J, 41 anos);

"Quando a doença é grave, vai no hospital, procura um medico né! Quando a doença é besta, toma o Chá" (M.P.J 43 anos).

Diversos fatores mantêm o uso de plantas medicinais, como uma prática ainda forte na comunidade. De acordo Resende \& Cocco (2002), o uso das práticas alternativas em saúde tem persistido, entre outros motivos, pela dificuldade no acesso à assistência de saúde para parte da população. Por seu baixo custo, as plantas medicinais representam, em muitos casos, a única alternativa possível para esta parcela da população (AZEVEDO \& SILVA, 2006). Percebe-se então, que o conhecimento referente às plantas medicinais é de grande importância para as comunidades tradicionais e precisa ser preservado para o benefício da humanidade.

\section{REFERÊNCIA}

AMOROZO, M. C. M.; PINTO, E. P. P.; FURLAN, A. Conhecimento popular sobre plantas medicinais em comunidades rurais de mata atlântica - Itacaré, BA, Brasil. Acta Botânica Brasílica, v. 20, n.4, p. 751-762, 2006.

AMOROZO, M. C. M. Uso e diversidade de plantas medicinais em Santo Antonio do Leverger, MT, Brasil. Acta Botânica Brasílica, v.16(2), p. 189-203, 2002.

ARAUJO, E. L. et al. Acanthospermum hispidum DC (Asteraceae): perspectives for a phytotherapeutic product. Revista Brasileira de Farmacognosia, João Pessoa, v. 18, 2008.

ARNOUS, A. H. Plantas medicinais de uso caseiro - conhecimento popular e interesse por cultivo comunitário. Revista Espaço para a Saúde, Londrina, v.6, n.2, p.1-6, 2005.

BAHIA. SEPLANTEC. Subsecretaria de Ciência e Tecnologia. Inventário de Plantas Medicinais do Estado da Bahia. Salvador, 1979.

AZEVEDO, S.K.S.; SILVA, I.M. Plantas medicinais e de uso religioso comercializadas em mercados e feiras livres no Rio de Janeiro, RJ, Brasil. Acta Botânica Brasílica, v.20, n.1, p. 185-194, 2006.

CEI, Centro de Estatísticas e Informações (BA). Informações Básicas dos Municípios baianos: Região Litoral Norte - Salvador, 1994.

FIDALGO, O. \& BONONI, V. L. R. Técnicas de coleta, 
preservação e herborização do material botânico. Instituto de Botânica, São Paulo, 1984.

FONSECA-KRUEL, V.S.; PEIXOTO, A.L. Etnobotânica na Reserva Extrativista Marinha de Arraial do Cabo, RJ, Brasil. Acta Botanica Brasílica, v.18, n.1, p. 177190, 2004.

FRANCO, E.A.P; BARROS, R.F.M. Uso e diversidade de plantas medicinais no Quilombo Olho D'água dos Pires, Esperantina, Piauí. Revista Brasileira de Plantas Medicinais, Botucatu, v.8, p. 78-88, 2006.

MONTELES, R. ; PINHEIRO, C.U.B. Plantas medicinais em um quilombo maranhense: uma perspectiva etnobotânica. Revista de Biologia e Ciências da Terra, v. 7, n.2 p. 38-48, 2007.

MOREIRA, R.C.T. et al.. Abordagem Etnobotânica acerca do Uso de Plantas Medicinais na Vila Cachoeira, Ilhéus, Bahia, Brasil. Acta Farmcéutica Bonaerense, v. 21, n. 3, p. 205-211, jun. 2002.
MOSCA, V.P.; LOIOLA M.I.B. Uso popular de plantas medicinais no Rio Grande do Norte, Nordeste do Brasil. Revista Caatinga, Mossoró, v.22, p.225-234, 2009.

PATZLAFF, R.G. Estudo Etnobotânico de plantas de uso medicinal e místico na comunidade da Capoeira Grande, Pedra de Guaratiba, Rio de Janeiro, RJ, Brasil. 2007. 147p. DISSERTAÇÃO - Instituto de Pesquisa Jardim Botânico do Rio de Janeiro. Rio de Janeiro.

PIRES, M.V. et al. Etnobotânica de terreiros de candomblé nos municípios de llhéus e Itabuna, Bahia, Brasil. Revista Brasileira de Biociências, Porto Alegre, v. 7, p. 3-8, 2009.

REZENDE, H.A.; COCCO, M.I.M. A utilização de fitoterapia no cotidiano de uma população rural. Revista da Escola de Enfermagem da USP, v.36, n.3, p. 282-288, 2002.

SARTÓRIO, M.L. et al. Cultivo Orgânico de Plantas Medicinais. Viçosa: Editora Aprenda Fácil, 2000, 258 p. 